\title{
11. Understanding Resilience and Reducing Future Vulnerabilities in Social-Ecological Systems
}

\author{
Brian Walker
}

I begin this contribution by examining what resilience means and then considering the implications for future vulnerabilities. The use of the term 'resilience' has risen markedly in recent years in response to growing awareness and uneasiness about looming shocks. They include global and regional financial crises, climate and weather shocks, pandemics, social unrest, regional wars and refugees, among others. These are rising in frequency. The frequency of such events worldwide is reminiscent of Winston Churchill's famous volume The Gathering Storm: we have that sense of the gathering storm and wonder what we can do. Will we be able to cope? That question is worrying many people, and that may suggest why we find the term resilience so much in use today. So, will we be able to cope?

Two recent surveys of the Australian media have checked thousands of sources for every occurrence of the word 'resilience' to look at what it meant, and they show how its use is rising and how very different are two common uses. The first one suggests the view that if authorities try to help people too much they do not bother to look after themselves, while the other is exactly opposite, expressing a hope that the concept of resilience does not erode into a justification for denying help to communities. So, there are two ends of a continuum about what to do and how much to help people. Nevertheless, this term has greatly different meanings in the minds of many people. In essence resilience is a framework for understanding how persistence and transformation coexist in living systems. In Chapter 5 of this volume, Peter Ho has talked about 'complex systems'; I call them 'living systems', but 'complex adaptive systems' is what they are.

Complicated systems and complex systems are different. Complicated systems are relatively simple in the sense that they have linear dynamics; they are entirely predictable. If you prod something and you know about it you can predict what will happen. Predictability is not possible in complex adaptive systems because they self-organise. Its parts themselves are capable of change. They can change their own behaviour and therefore they can adopt different things; their unpredictability has to be taken into account. I was told of a wonderful example of trying to enhance salmon fisheries in British Columbia where the industry thought: 'If we could just get more young salmon down to the sea then more salmon would come back to spawn and we would have more salmon.' So they enhanced production in the nurseries and put lots of young salmon in the rivers 
that went down to the sea; but on the way down to the sea these fingerlings went past another type of fish that had never eaten salmon because they ate other things. But when an enormous volume of little salmon fortuitously came by they switched their predation behaviour and ate the salmon, so fewer salmon actually made it to the sea. A complex system had become totally unpredictable. Complexity is a change in the behaviour of the system because the system selforganises in response to changing circumstances.

So the definition of resilience is the capacity to absorb disturbance, to reorganise so as to retain essentially the same function, structure and feedbacks, to have the same identity. 'Identity' is what brings the world of psychologists and the world of ecologists together. Psychologists talk about people losing their identity when they go into a catatonic state or shift mentally, while we talk about ecosystems having a different identity - they change from one kind of operational system to another, not just another phase of the same system.

In layperson's terms, resilience is the ability to cope with shocks and to keep functioning in a similar way. The addition of 'feedbacks' is a key idea because the feedbacks in a complex system determine its self-organising capacity. The Resilience Alliance ${ }^{1}$ has compiled a database of what we call 'threshold shifts' or 'phase shifts' in systems. In each case where we have been able to gather enough data we have identified a threshold crossing that shows a change in a critical feedback; so understanding the feedbacks, especially across domains and scales, is an essential part of resilience thinking.

Resilience has three critical components. There is 'specified resilience': the resilience of one thing relative to another, which has to do with threshold effects. Then there is 'general resilience', where no particular part of the system or particular shock is identified because it has more to do with the total adaptive capacity of the system. And third, there is 'transformability', the capacity for transformational change to a different kind of system. I will now deal with each of those components to deepen our understanding of the way resilience can operate.

\section{Specified Resilience}

Specified resilience is about thresholds or tipping points or critical transitions they are referred to in many different ways. The commonly assumed response of the equilibrium or stable state of a system to a change in its controlling variables suggests no threshold effect, as shown in Figure 11.1 (a). Its trajectory might be curvilinear or linear but the change is continuous. In fact, there are four kinds

1 The Resilience Alliance is a research organisation of scientists and practitioners from various academic disciplines exploring the dynamics of social-ecological systems. See $<$ www.resalliance.org $>$. 
of possible responses, as shown in Figure 11.1, including a step change in (b) and two nonlinear types of change in (c) and (d). These involve what are known as hysteretic effects. In these two cases, as you increase a controlling variable the stable state of this system moves gradually and then jumps up. If you then decrease the controlling variable it does not drop down at the same point as it jumped up. It needs to decrease a long way further down. The crucial point here is that this allows alternative stable states for the same amount of the controlling variable. So the state of the system can exist in either of two states for the same amount of the controls on the system.

(a) No threshold effect

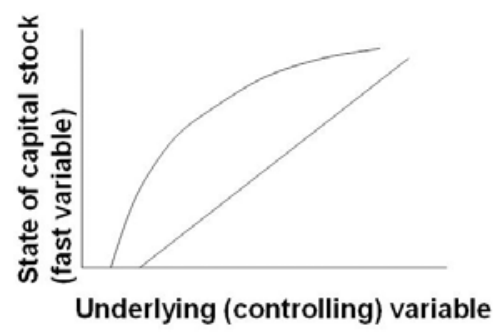

(c) threshold, alternate stable states

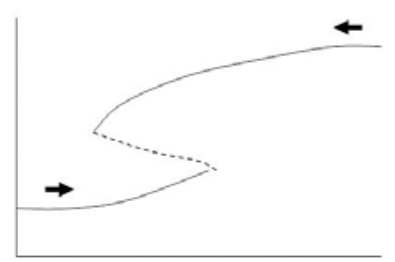

(b) threshold, no alternate attractors

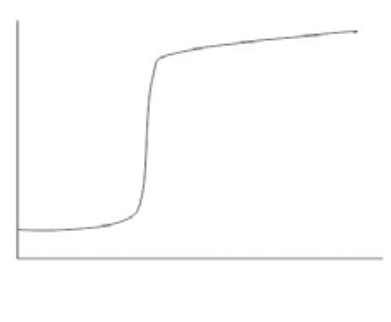

(d) irreversible threshold change

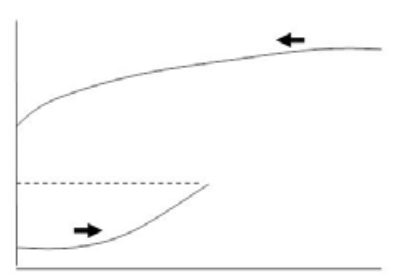

\section{Figure 11.1 Kinds of Thresholds (Tipping Points)}

Source: Author's summary.

An example of a step change is a landscape where the controlling variable is the percentage in its natural habitat, and the state of the system we are interested in is the number of fauna species that persist there. For instance, if one were to start clearing a landscape the fauna would gradually decline but then suffer a sudden drop. Many published papers from different parts of the world show that about 30 per cent landscape cover is a critical level (it varies depending on the pattern and the area, but there is a sudden, critical change at some level). There is another one at about 5 per cent so there are a number of step changes. It is not a smooth change.

An example of the kind of changes in Figure 11.1 (c) is algal blooms in lakes, where the lake suddenly becomes eutrophic (full of algae and foul smelling). 
The controlling variable is phosphate in the sediment at the bottom of the lake. The state of the system is the amount of phosphorous in the water, which determines the amount of algae. The chemistry is quite complicated, but it has to do with the solubility of phosphate under different levels of oxygen. As the algae increase, they die and then sink to the bottom where oxygen is used up in decomposing them. When there is no oxygen in the water phosphorous becomes very soluble and is released from the sedimentary mud in large quantities and the algae numbers suddenly shoot up. The amounts of phosphorous in the water and sediment have to be brought way down before water phosphorous (and therefore algae) can return to the pre-jump level.

The fourth kind, in Figure 11.1 (d), is an irreversible threshold, where the system never comes back to the pre-jump level. An example of this is topsoil salinity. Catchment salinity is a big problem in Australia and the controlling variable is the depth of the water table below the surface; salt in the topsoil is the state of the system. As the water table rises, because of clearing the landscape, it brings salt with it. When the water table reaches a critical levelabout $2 \mathrm{~m}$ below the surface for most soils - capillary action pulls the water up to the surface and brings the salt up with it. Salt disperses clay-making it soapy - and the water will not seep back down again. After the water table drops again, it takes a great deal of rainfall to eventually leach the salt down, so the topsoil stays salty. Effectively once the system passes that threshold it is in an irreversible salinised state.

All four of these kinds of thresholds or tipping points occur in all kinds of systems. Fortunately, many of them are smooth, as in Figure 11.1 (a). But if any of the other three occurs in a system it is important to know about them because they define the critical boundaries within which that system has to operate- or, if not, it will soon look very different and have a different identity.

The thresholds I have described exist in ecological systems but they also occur in social systems. In economic systems there is a debt-to-income ratio that everyone knows about; it has a very strong threshold effect and once you increase debt beyond a threshold level it is very difficult to get below that debtto-income ratio again. There are many examples of research on threshold effects of behaviour in crowds, such as riots; also in fashions and fads where they suddenly take off. Some systems do not have a hysteretic effect in the return path but crowd behaviour does. For example, with a rioting crowd if the threshold level of rioters is below a certain number the riot dies away if the provocation ceases; however, once over the threshold (seemingly around 20 per cent), the riot takes off and continues even after the provocation is removed.

Social, ecological and biophysical systems exhibit nonlinear threshold behaviour, which results in alternating stable states of systems (or alternating stability 
domains). One could ask: what variables reached threshold levels or tipping points in the 'Arab Spring' political outbreaks in the Middle East recently? What was building up? One proposition may be that the threshold was brought much lower than before due to social media: mobile phones enabled it to take off at a much lower level of provocation than would have been required earlier. This is an interesting idea because it reinforces one of the next three points I want to stress.

First, a threshold occurs where there is a change in feedbacks, and feedback effects can be counterintuitive; they can be quite puzzling, as illustrated by the following example.

Salvinia molesta is a weed from South America that filled lakes in northern Queensland. After some tests, a little weevil (Cyrtobagous salviniae) was introduced from South America (where salvinia originates), which eats only salvinia, and it cleared the lake. Papua New Guinea also has lakes with the same salvinia problem and when the weevil was introduced there nothing happened. An Australian scientist studied this and discovered that the weeds actually needed to be fertilised to grow even more. What he found was that the protein level in the weeds was just below the critical amount needed for the weevil to breed; the protein level had to be raised. Nitrogen fertiliser was added; the protein level went up and the weevils took off and bred and controlled the weed. What was interesting is that once that had happened and the weevil numbers increased above a threshold number of their own, the effect of grazing on the weed was to increase its protein level, and so it never needed to be fertilised again as long as the weevil numbers remained high enough to control the salvinia.

The second point is that thresholds interact and can move-they are not necessarily constant. As an example, coral reefs can be healthy or stressed. They can exist in the normal coral state as well as a macro-algal state, and various others, the worst being slime. The two controlling variables determining these states are the amount of fishing, particularly of herbivorous fish, and extra nutrients flowing onto the reef. The nutrients promote algae, while grazing by herbivorous fish reduces algae, so if you go above a certain threshold of fishing and extra nutrients the reef goes from the coral state to the macro-algal state. Even if the fishing is reintroduced, it stays in the algal state unless something else intervenes that can get rid of the algae.

A point I underline in this example is that the threshold level is not fixed. Climate change has an effect on the threshold positions. The threshold is lowered by climate change, carbon dioxide and the acidification of the water. So the effect of climate change is to reduce the resilience of the reef to fishing and nutrient inflow. It will take less fishing pressure and fewer nutrients to flip a coral reef into the algal phase as climate change increases because that threshold, like most, is not fixed but can shift and change. 
The third point about thresholds is that they occur across scales and domains that can have cascading effects; by domains I mean the ecological, the economic and the social subsystems. In the Goulburn-Broken catchment in south-eastern Australia 10 thresholds were identified by researchers, together with the local people, that occurred at three spatial scales - farm to landscape scale, landscape to catchment, state to nation-and in three domains: the social, the economic and the biophysical. These 10 thresholds have cascading effects. So once a threshold is crossed it can cause the crossing of another threshold or it can reduce the likelihood of crossing another threshold.

So from a resilience perspective, a linked social-ecological system is viewed as a set of interacting thresholds across domains and across scales, subjected to external shocks - price shocks, changes in markets, climate, diseases and so on-and, depending on the shock, one or more of these thresholds are likely to be crossed, which can set in chain a cascading effect. That represents a different way of thinking about systems other than trying to optimise some particular part of them.

\section{General Resilience}

General resilience, involving adaptability, is the capacity of all parts of the system to cope with many types of shocks. Many different studies show that having high diversity, and especially what we call response diversity, is important for conferring general resilience. It is often mistakenly called redundancy. In systems where economic efficiency is important redundancy means where there are two ways of doing something it is preferable to get rid of one and keep only the one that is doing the job best. Scientists do not see that as undesirable redundancy. Rather, they say if a system has several elements that do the same thing but in somewhat different ways, that confers response diversity. If one element is knocked out the other one can begin to do it. So if five species in a forest can fix nitrogen and something happens to one of them the other four can take up the slack. If there is only one legume species (nitrogen fixer) and a disease wipes it out the whole function of nitrogen fixation will be lost.

Being modular in structure and not overly connected is another contributor to general resilience. An overly connected system means problems and diseases can be transmitted rapidly through the system as a whole, while having no connections at all implies isolation. A modular system indicates enough connectedness to exclude isolation, but not so many connections that defences collapse.

General resilience is enhanced by the ability to adapt quickly and effectively to change. Having tight feedbacks is one aspect of this: when the length of 
contributing components in a feedback loop increases, it decreases the resilience. If it takes six steps instead of three to record and respond to something, resilience is lost. Also, being open, allowing immigration and emigration (ecologically or socially) enhances resilience. And having reserves is necessary, whether physical reserves like seed banks or social reserves like memory. Several years ago when the massive tsunamis hit South-East Asia, those communities which did best had old people nearby who remembered that when the water goes out people should run away from the water. Such social memories conferred resilience.

Other important qualities of general resilience applying particularly to human systems are fostering innovation and novelty/experimentation. Social systems are more resilient when we promote innovation and experimentation, but so much of the way our systems work reduces the potential of these attributes.

The final attribute I will mention is having overlapping institutions and polycentric governance. We are talking here of building social capital through leadership, trust and strong integrated networks. The work of Elinor Ostrom has shown how they enable long-term persistence, as opposed to having highly efficient, non-overlapping and single systems of governance. Ostrom, as a founding member of our Resilience Alliance, found some wonderful stories about small, long-term irrigation and forestry systems that have persisted for 500 years or more. What rules enabled that system to persist over time? These rules are incredibly interesting. Ostrom (1990) identified boundary conditions of the rules that have to be in place. What happened with many of them is that they evolved under a variable, uncertain environment and coped. Spanish agriculture (a persistent system) has now been opened up to the European Union. Spaniards now must allow cheaper oranges from elsewhere into their region and they can sell their water outside their region. This has entirely changed the rules that enabled that system to persist - globalisation has disrupted the rules. We still have to think of it as a complex adaptive system and also need now to think: 'How is the world changing?' Or, to put it differently: 'What are the rules for changing the rules?' Rules are going to change, so the issue is whether we can put in place rules to change our own rules so that we can transform through time instead of becoming an economic basket case.

I will now emphasise four additional points about general resilience. First, resilience is not about avoiding change. Trying to keep a system constant reduces resilience. If you never burn a forest it becomes increasingly prone to dying as a result of fire. The only way to make a forest resilient to fire is to burn it occasionally because otherwise the species capable of coping with fire will be outcompeted, will disappear and the forest will lose the ability to handle a fire. 
Next, making a system very resilient in one way at one spatial scale can cause it to lose resilience in other ways or at other scales. There is a trade-off. A socialecological system cannot be understood or managed at only one scale; there are trade-offs when resilience is applied in practice.

The third point is that general resilience is neither good nor bad. Often people talk about resilience as obviously good; however, undesirable states of systems can be very resilient, such as dictatorships and saline landscapes. Resilience is a property of a system. A very resilient 'bad' state is incredibly difficult to deal with and a system that was once considered desirable can become very undesirable through changes in external conditions to which it has adapted.

And finally, most losses in resilience are the unintended consequences of narrowly focused optimisation, mostly stemming from failure to recognise crossscale and cross-domain feedbacks.

It comes down to the trade-off between specified and general resiliencies, which are both important. The 'rule of hand' states that at any one scale there are no more than three to five controlling variables that are really important at any time. If there were many variables the system would exhibit chaotic dynamics and wouldn't persist over time. It would eventually degrade until it became a simpler system. The handful of really important controlling variables defines the boundary conditions that limit the system so it keeps functioning as it is. It is necessary to understand what those variables are and that's where you should put your money. The way to get at those is to do a 'feedbacks' kind of analysis. You need to think that if you change this then what feedback loops follow? What controls the dynamics when you change any one part of it? This eventually simplifies down to a handful of controlling variables that define the axes of the system in which its behaviour remains. If, however, you put all your money on one variable, saying 'water depth is really an important controlling thing so we should put all of our money into controlling that, keeping it below that threshold', and if in doing that something else that is important is changed and you haven't really thought about it, you could come unstuck. You might end up with a massive disease outbreak because of a consequence of that action that had nothing to do with water depth. So you must constantly bear in mind general resilience. How do you remain generally resilient when you cannot predict what shock might occur and what the uncertainties are, and where the possibility of transformation is?

So although there is some trade-off between general and specified resiliencies you can prioritise because in each case you can identify where the weaknesses are. I run resilience assessment workshops with catchment management authorities and I find the easiest issue to come to grips with when they start working on specified resilience is to identify where the thresholds are. We use a process called state and transition models, which ask: 'What states can the system be 
in?' It can be in one of several states. This requires another question: 'How does it get from one to the other?' When you ask those questions people bring their knowledge and indicate what needs to happen for it to go from one state to another. So you can start by looking for a threshold level to get to grips with a particular part of the system. For general resilience, however, it is much fuzzier.

\section{Transformability}

Being able to adapt and being generally resilient will undoubtedly be very important in what we do in the future for our society, for ecosystems and for social-ecological systems. But when does further adaptation simply amount to digging the hole deeper? The first rule about holes is that when you are in one, stop digging. Getting past the state of denial is not easy. I have heard irrigation farmers in areas of the country that are drying out say: 'No, we just need to get a bit more efficient.' Their adaptation is to dig the hole deeper, making it much harder to shift to something else, as they probably will have to do.

So, if a shift into a bad state has occurred or if one clearly is going to happen then the only option is transformation, which is the third big property or concept of resilience: the capacity to become a different kind of system, a new way of living or making a living. This is not just another phase of the same system but a system that is defined differently.

In most of the world today the rates of change (social, ecological and climatic) demand a process of continuous transformation, not adaptation; that is a deeply uncomfortable idea because most people hate fundamental change. Adaptation people can do, though it is hard, but they hate fundamental change. So if communities are told they can no longer be irrigation farming producers, but must do something else, they will fight tooth and nail. Yet fundamental change is being demanded of us because the rates of change occurring in the world today require it.

Transformability has three determinants. The first is preparedness to changegetting beyond the state of denial is the most difficult stage. The second determinant is options for change. What new trajectories are possible? This emerges from support for experiments, for novelty, for continual learning to identify the options. The third is the capacity to change; even if a good option can be identified can it be adopted? The capacity to change is largely about the levels of capital: human, financial, knowledge and natural capital and higher-scale support, such as appropriate institutions. Higher-scale support includes governance, but governance, which is so important, is often lacking or disappointing. 
So if we look at the options for change it is especially important to support creativity and identify new options. An idea that comes out of transition theory in The Netherlands is about 'safe arenas'. A group of social scientists is trying to transform five stressed policy areas in The Netherlands. They say The Netherlands cannot keep doing what it is doing in terms of energy, transport, old age, agriculture and water. The country has to change fundamentally. To change energy in The Netherlands requires getting all the stakeholders together, including the major energy company, which is likely to prevent anything it dislikes because it is the big, powerful player. So this group of social scientists proposes creating safe arenas for experimentation that are protected from the dominant regime, allowing people to do unusual things. Many of those will fail, but those that succeed will start to become mainstream and then you will get a trajectory shift.

It is also important to encourage change rather than help prevent change. All too often help from government seems to be help to keep doing the same thing. When people want to do something different they are told: 'Oh no, you can't use money for that. The money's dedicated to do this and you can't use it to do that.' So government effectively prevents communities from doing anything novel.

Resilience and transformability are not opposites. In Australia's MurrayDarling Basin it is now clear there is not enough water in the system to meet the water allocations communities were originally given (even in a wet period), so in order for the whole basin to support viable, sustainable, long-term farming communities with high wellbeing, parts of the system have to transform. Not all of the existing communities can keep doing what they are doing - and of course the tough question is: 'Who has to change?'

The questions that apply to these communities, spatial areas and even nations are: 'Where is there a need to build the resilience, and where is there a need for transformational change?' If as a society we can distinguish between adaptation and transformation, and try not to build resilience everywhere, we will make considerable progress forward.

Almost every time we have done the exercise the weakest part is in the governance. Achieving transformational change depends on the kind and strength of governance that is in place. I keep coming back to that. Often, those responsible for it are the root of the problem. I met one very perceptive person working in one of the catchment management authorities who was complaining about the intransigence of the levels above her-and many of them were intransigent. She then said perceptively: 'But hang on: you know, when we get the power to do something we don't want to distribute it down either, we want to keep control.' There is a kind of urge at every level to keep control and power, to consolidate power and not to distribute it. A distributive governance 
system allows a decision to be made at the level at which it is most appropriate. That level may change over time and depending on context. Under certain circumstances you should make it at a higher level, at other circumstances at a lower level; but keeping it always fixed is not going to work and will be particularly consolidating because then there is the natural tendency for control to creep upwards.

Current global governance efforts are largely failing because national selfinterest and the silo structure of international agencies suppress and mask the secondary feedback effects of sub-global activities. This issue is of great concern to people in the Beijer Institute in Sweden and the Stockholm Resilience Centre, where we have been trying to look at how the planet is functioning at the moment. We see as a major problem the inability of international institutions and agencies to connect in a collaborative, self-organising way. Changing the planet's current unsustainable trajectory will require a process of continuous transformational change, not adaptation, and a new social contract; and that needs to be informed by a resilience perspective of what is happening.

So, to conclude: how do we operationalise a resilience perspective? Well, there is no single recipe. Applying resilience amounts to putting a resilience lens over what is already being done and whatever planning or management frameworks are being used. This is the challenge ahead.

\section{Reference}

Ostrom, E. 1990. Governing the Commons: The Evolution of Institutions for Collective Action (Cambridge: Cambridge University Press). 
This text taken from Future-Proofing the State: Managing Risks, Responding to Crises and Building Resilience, edited by Jonathan Boston, John Wanna, Vic Lipski and Justin Pritchard, published May 2014 by ANU Press, The Australian National University, Canberra, Australia. 